\title{
Characteristics of Adverse Effects When Using High Dose Short Term Steroid Regimen
}

\author{
Kyung Hyun Min, Chung-Ku Rhee, Jae Yun Jung and Myung-Whan Suh \\ Department of Otorhinolaryngology, Dankook University College of Medicine, Cheonan, Korea
}

\author{
Received July 26, 2012 \\ Revised August 25, 2012 \\ Accepted August 31, 2012
}

\section{Address for correspondence Myung-Whan Suh, MD \\ Department of Otorhinolaryngology, Dankook University \\ College of Medicine, \\ 201 Manghyang-ro, Dongnam-gu, \\ Cheonan 330-715, Korea \\ Tel +82-41-550-3974 \\ Fax +82-41-556-1090 \\ E-mail drmung@naver.com}

Background and Objectives: Although the adverse effect of long term steroid usage is well known, the adverse effect of short term high dose steroid usage has not been studied thoroughly. The purpose of this study was to identify the characteristics of the adverse effects when using a high dose steroid for a short term. We also compared the adverse effect of steroid between in- and out-patients. Subjects and Methods: The medical record of 500 patients, who were treated with methylprednisolone $48 \mathrm{mg} /$ day for 1-2 weeks, was retrospectively reviewed. Steroid was prescribed due to SSNHL, Bell's palsy, Ramsay-Hunt syndrome and herpes zoster oticus. The incidence of each adverse effect was analyzed, and also the time point of detection was analyzed. The 500 patients were composed of 250 in-patients and 250 out-patients. The adverse effect was compared between these two groups, as well. Results: Adverse effect of steroid was found in $33.0 \%$ of the patients. Among these adverse effects, abdominal discomfort (26.8\%) was most common, followed by skin rash (14.7\%), swelling (13.4\%), and hot flush (6.9\%). Abdominal discomfort and hot flush was mostly detected on the first week. Meanwhile, the skin rash was usually detected on the third week. The incidence of adverse effect was significantly higher in the out-patient group. Conclusions: The incidence of high dose short term steroid treatment may be very high. The patients should be warned about these adverse effects. Monitoring should be performed until 3-4 week, since some adverse effects may not be detected until this time point.

Korean J Audiol 2012;16:65-70

\section{Introduction}

Steroids are frequently used drugs for the treatments of sudden hearing loss and facial palsy, known to have effectiveness of anti-inflammation and immunosuppression, when used at high-doses. ${ }^{1)}$ The mechanism for steroid in sudden hearing loss to express the effectiveness in its treatment has still been unknown accurately, but assumed to reduce the inflammation of cochlea and acoustic nerve. ${ }^{2)}$ Also, steroid has been known as preventing autonomic disturbance incurring due to denervation of facial nerve in patients with facial palsy, and also preventing the progression of incomplete paralysis to complete paralysis. ${ }^{2}$ In spite of its positive effectiveness, long term use of steroid can cause adverse effects, including osteoporosis, renal impairment, infection, gastrointestinal disorder, depression, hypertension and diabetes. ${ }^{3)}$ Aforementioned adverse effects incur when using steroid over a long time in general. But ear nose throat (ENT) practice uses high-dose steroid over a short time more frequently than a long time. Meaning, the adverse effects of steroid that ENT doctors may experience during actual medical care is possibly different from the description contained in the drug information. There are not so many cases identified in terms of potential adverse effects, and their prevalence from the use of a short term high-dose steroid even in the literatures. According to a report, the use of a short-term steroid therapy in children with sudden hearing loss, it reported on the incidences of nasal bleeding, hepatic impairment, acne, gastroenteritis and rump abscess after using steroid. ${ }^{4}$ However, these reports had focused on nothing but listing the phenomena, while treating children rather than focusing on the adverse effects of steroid. There are little reports on the types of adverse effects and the prevalence of each adverse effect likely to appear when prescribed high-dose steroid for 1 to 2 weeks in patients with sudden hearing loss or facial palsy 
who were usually healthy. To this end, this study aimed to investigate the types, prevalence and occurrence time of adverse effects when used a short-term high-dose steroid therapy and to identify whether there were any difference in the prevalence of adverse effects between those who received in-patient treatment opposed to those who received out-patient treatment.

\section{Subjects and Methods}

The study was conducted according to the principles of the Declaration of Helsinki and to good clinical practice guidelines. Medical records of 500 patients were retrospectively analyzed, who had received high-dose steroid therapy, either as in-patient or from out-patient prescription, from January 2008 to December 2011. The underlying reasons for using steroid had included sudden sensorineural hearing loss, Bell's palsy, Ramsay-Hunt syndrome and herpes zoster oticus. Herpes zoster oticus, described in this study, denotes herpes zoster incurred in the ear without accompanying facial palsy. In this study, the schedule of steroid use was to administer methyl prednisolone for 1 week at dose of $48 \mathrm{mg} /$ day, followed by reducing 10 $\mathrm{mg}$ daily (intakes of 12 days in total). In order to control the errors that adversely effects patients but they failed to accurately inform the applicable details to medical staff, all involved patients were fully informed of the adverse effects that were likely to appear prior to the use of the drug, and received a list of adverse effects (Fig. 1). In addition, at every visit for an outpatient medical care, from 0.5 to 2 times weekly, the onset of adverse effects had been confirmed in patients; thus, this study was conducted as having those patients with accurate description contained in the records for the presence/absence of adverse effects as the study subjects. Although the study was designed as a retrospective structure, it was ensured not to be confused between "adverse effects not checked" and "no adverse effects" by taking aforementioned measures.

The abdominal discomforts included all disturbances in the gastrointestinal system, such as abdominal distention and dyspepsia. Skin rash included all rashes from local to systemic rashes (Fig. 2), but without including small number of rashes like 1-2 spots or pimples. Subjective feelings of swelling face or extremities were included as edema. Hot flush had included such symptoms like facial blush or sense of fever. Overall dyspepsia included those cases that patients feel systemic powerlessness or feel losing vitality. Other adverse effects, except skin rash were defined based on the subjective feelings of patient rather than any objective signs. Meaning, some patients complained of discomfort subjectively, but there were cases without a clear abnormality when medical staff evaluated such discomfort.

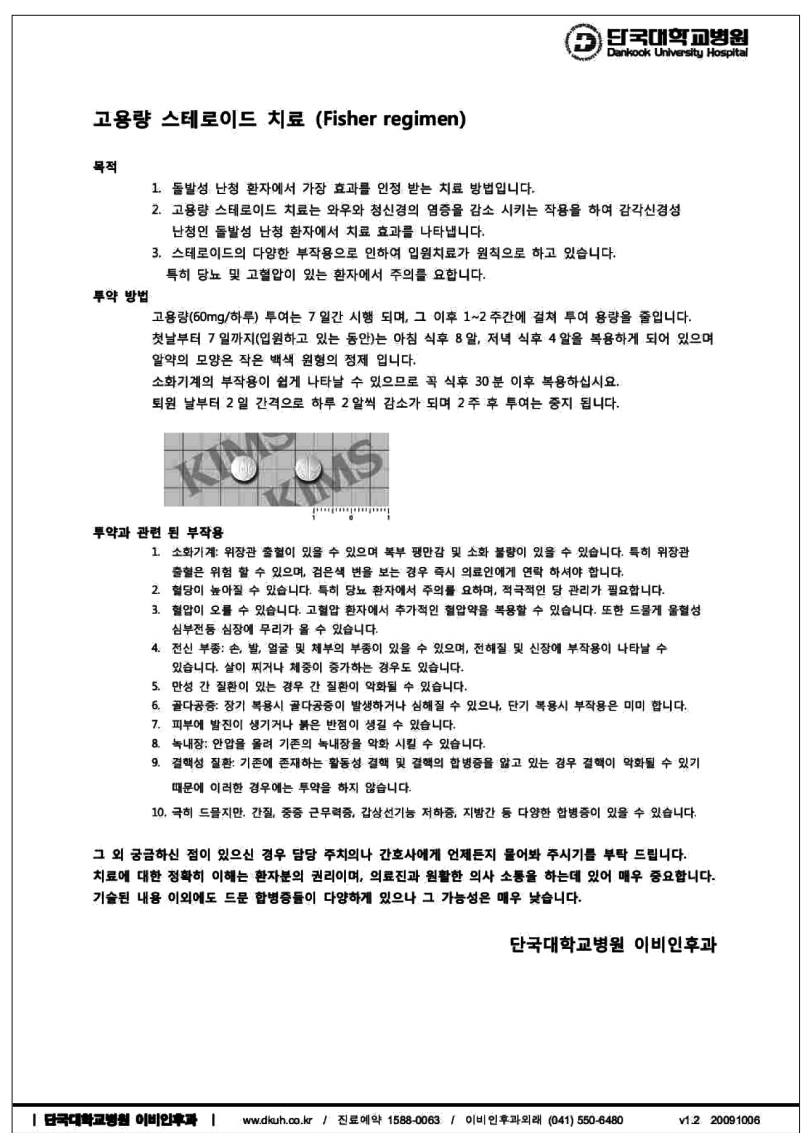

Fig. 1. List of adverse effects known to be related with systemic steroid treatment. All patients involved in this study were fully informed about adverse effects likely to appear in prior to using the drug, and received the list of adverse effects.

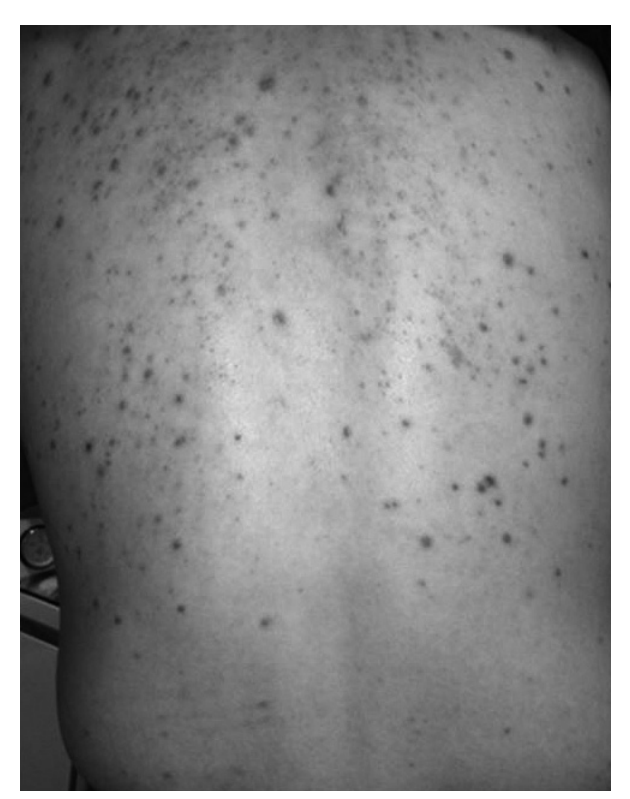

Fig. 2. Example of skin rash induced by high dose steroid therapy. Methylprednisolone $48 \mathrm{mg}$ was prescribed for 1 week with 5 days of tapering dose due to sudden sensorineural hearing loss. After several weeks multiple red papules developed over the whole body. It was especially noticeable on the back of this patient. 
Out of the 500 patients in total, 424 patients (84.8\%) were with sudden sensorineural hearing loss, followed by 49 patients $(9.8 \%)$ with Bell's palsy, 13 patients $(2.6 \%)$ with RamsyHunt syndorme and 14 patients $(2.8 \%)$ with herpes zoster oticus. The mean age of patients was $45.7 \pm 17.0$ years old and the gender ratio was $278: 222$. The numbers of those treated as in-patient and those treated as out-patient were 250 and 250 patients, respectively. The out-patient group and the in-patient group were constituted with each 250 patients intentionally, taking age and gender into account. The mean age of patients was $46.5 \pm 17.2$ years old in the in-patient group and $45.0 \pm$ 16.8 years old in the out-patient group, and each applicable gender ratio was $137: 113$ in the in-patient group; whereas, it was $141: 109$ in the out-patient group. There was no significant difference in the mean age, as well as the gender ratio between the two groups. Hospital admission was determined by personal choice of patients, as well as depending on the overall health conditions, and the in-patient duration was 7 days. There was no difference in steroid prescription method and dosage between the in-patient group and the out-patient group. However, there were differences in the administration of digestives, blood circulation promoter and anti-viral agent other than digestives. More treatments with aforementioned drugs were implemented in the in-patient group additionally, and as the route of administration, injection was used more than the oral form.

First of all, this study had investigated the types, prevalence and onset timing of adverse effects after the intake of steroid in the entire patient group. The onset timing of adverse effects was calculated based on the time point that the applicable symptoms were found for the first time. Thereafter, the study had performed a comparative analysis to identify whether there was any difference in the prevalence of adverse effects between the in-patient group and the out-patient group. Computer software (SPSS v12.0, IBM, Armonk, NY, USA) was used for statistical analysis with Student's t-test and chi-square test.

\section{Results}

\section{Total patient population}

The incurrences of adverse effects were confirmed in 165 patients $(33.0 \%)$ out of 500 patients in total, who had received steroid therapy. Among those adverse effects, 62 patients (26.8\%) had shown abdominal discomfort as the most, followed by 34 patients $(14.7 \%)$ with skin rash, 31 patients $(13.4 \%)$ with edema, and 16 patients $(6.9 \%)$ with hot flushes in order (Fig. 3). Abdominal discomfort and hot flushes had the highest prevalence in the first week, but were reduced in the prevalence at week 2 to 4 ; whereas, edema presented the highest prevalence at week 2, and skin rash at week 3 (Fig. 4). Rarely,

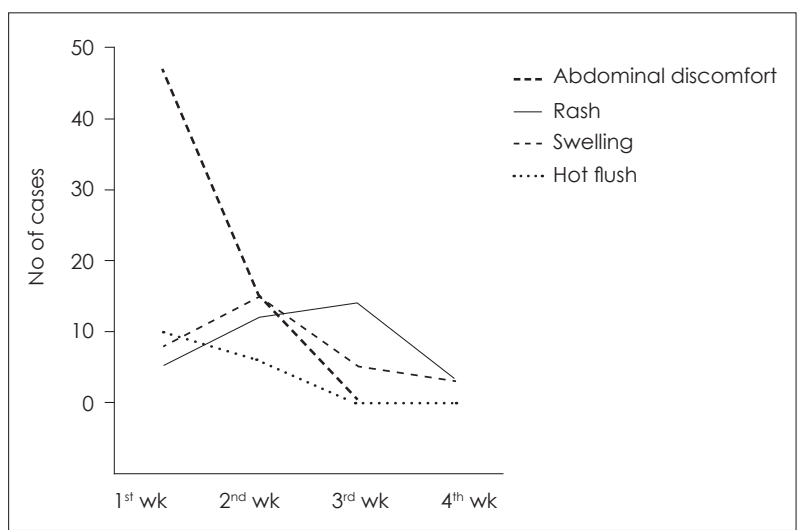

Fig. 4. Detection time point of each adverse effect. Abdominal discomfort and hot flush was mostly detected on the first week. Swelling was mostly detected on the second week. Meanwhile skin rash was mostly detected on the third week. Considering that steroid was only prescribed for 12 days, this means that adverse effects such as skin rash may develop 1-2 week after finishing the medication.
Fig. 3. Incidence of each adverse effect due to high dose steroid. When all the subjects were analyzed as a whole group, adverse effect of steroid was found in $33.0 \%$ of the patients. Among these adverse effects, abdominal discomfort $(26.8 \%)$ was most common followed by skin rash $(14.7 \%)$, swelling (13.4\%), and hot flush $(6.9 \%)$.

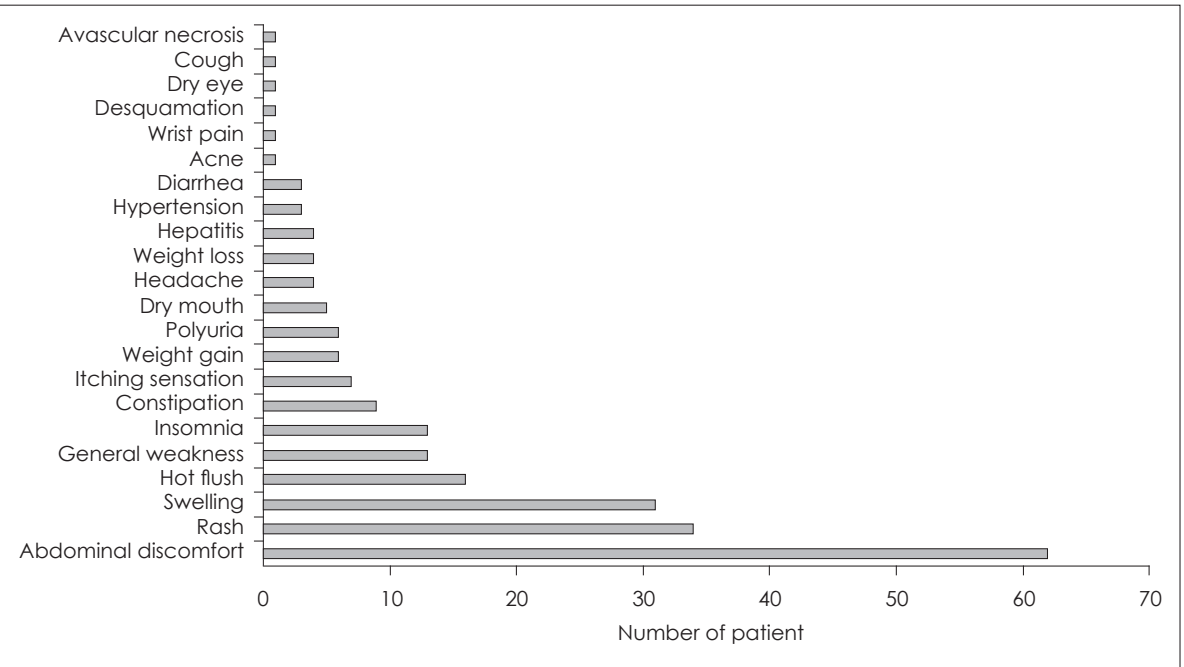



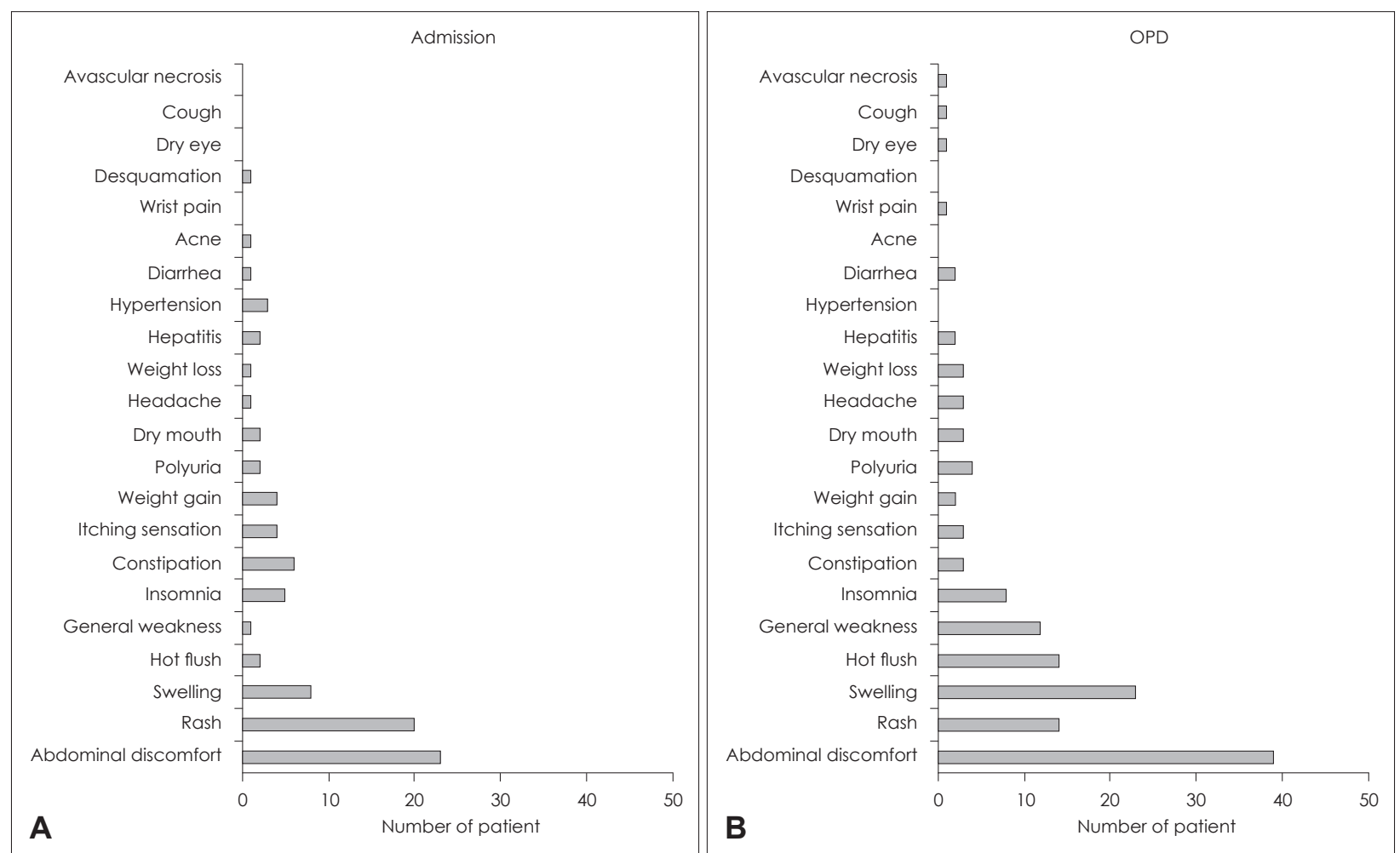

Fig. 5. Incidence of each adverse effect according to whether the patient was admitted or not. The incidence of each adverse effect was significantly different between the admission group (A) and the out-patient department (OPD) group (B). Abdominal discomfort and swelling were much more common among the OPD group when compared to that of the admission group. Meanwhile incidence of skin rash was lower in the OPD group.

but possibly, there were patients to whom serious adverse effects had incurred, such as avascular necrosis of hip bone $(\mathrm{n}=$ 1) and toxic hepatitis $(n=4)$.

\section{Comparison between the in-patient group and the out-patient group}

There were significant difference in diagnosis of the in-patient group and the out-patient group $(p=0.011)$. Sudden hearing loss was diagnosed in 200 in-patients $(80 \%)$ and in 224 outpatients (89\%), showing it was more in the out-patient group. Whereas, Bell's palsy were found in 30 in-patients (12\%) and 19 out-patients (8\%), 11 in-patients (4\%) and 2 out-patients (1\%) as Ramsay-Hunt syndrome, and 9 in-patients (4\%) and 5 out-patients $(2 \%)$ as herpes zoster oticus, presenting there were more numbers of in-patients in each diagnosis.

Incidences of adverse effects had been identified in 62 patients $(25 \%)$ out of 250 in-patients and in 103 patients (41\%) out of 250 out-patients. The prevalence of adverse effects was significantly lower in the in-patients compared to the out-patients $(p<0.001)$. Also, there were significant differences in the types of adverse effects between the in-patient group and the out-patient group ( $p=0.022)$ (Fig. 5). The adverse effects incurred from the in-patients were abdominal discomfort from
23 patients, skin rash from 20 patients, edema from 8 patients and constipation from 6 patients, in order; whereas, the adverse effects incurred from the out-patients were abdominal discomfort from 39 patients, edema from 23 patients, skin rash from 14 patients and hot flush from 14 patients, in order.

\section{Discussion}

From the study results, it was identifiable that the percentage of patients who had experienced adverse effects, among the total population of patients who has been treated by a shortterm high-dose steroid, was $33.0 \%$, indicating a quite high rate. Therefore, the information on adverse effects should be explained to patients before making the steroid prescription. This would reduce the anxiety of patients, prevent the distrust on the medical staff and increase the compliance to the treatment, even if any adverse effects incur. However, such values included non-specific and minor subjective symptoms complained by the patient in all, and the prevalence of clinically significant adverse effects was likely lower. Another fortunate fact was that the most adverse effects were not serious and recoverable after discontinuity of drug administration.

The interesting matter at the incidence of each adverse ef- 
fect is that some adverse effects begin to appear concurrently with the drug intake, whereas some adverse effects incur belated after completion of the drug intake. For example, abdominal discomfort had begun within 1 week immediately after taking the drug in many cases, while there was no case of having abdominal discomfort newly at week 3 when the drug administration was ended. Meanwhile, the most cases of skin rash had begun at week 3 , when the drug administration was complete. This was assumed because it took about 1 to 2 weeks until the skin reaction appeared, as the immune mechanism acted. Given the fact that the drug administration period was 12 days, it indicated these adverse effects could incur even 1 to 2 weeks after the completion of drug intake. Meaning, because there is a possibility to have belated onset of aforementioned adverse effects even after the completion of drug taking, it would be better to continue the observation of patients up to 3 to 4 weeks.

According to an existing report, adverse effects had been found in patients who took steroid after kidney transplant, such as hypertension $(15 \%)$, diabetes $(10 \%)$, fracture $(2 \%)$, avascular necrosis of hip bone (8\%) and cataract (22\%). ${ }^{5)}$ However, this study had inconsistency in the doses of steroid administration and the follow-up observation time for patients. It had limitations in making comparison of a certain dose-induced adverse effects, since some patient had used the shortterm therapy, while some other patients had used the long-term therapy. A study pointed out that steroid can cause adrenal suppression in patients with severe chronic obstructive pulmonary disease. ${ }^{6}$ Another study had reported that when performed the steroid therapy in 75 patients with polymyalgia rheumatica or giant cell arteritis, adverse effects had incurred, such as fracture (10 patients), dyspepsia (5 patients), gastric ulcer (4 patients), diabetes (4 patients) and cataract (2 patients). ${ }^{7)}$ This study was performed in patients who had been treated with prednisolone at the dose of $10-40 \mathrm{mg} /$ day for over 18 months, and the doses were properly increased or decreased, depending on the systemic conditions of patients. The time that had confirmed the onset of adverse effects was 1 time in 2 weeks for the first 2 months, and thereafter 1 time in 2-3 months. As aforementioned, the existing reports had focused on the adverse effects caused by the long-term steroid therapy, so it had difficulties in comparing with the results of this study. Nevertheless, the important difference, identified from the existing reports, and this study focused on the adverse effects predicted for a short-term prescription, which were mostly minor and recoverable, but the adverse effects predicted for a long-term prescription were more severe and harmful to the health.

When a simple comparison was performed between the inpatient group and the out-patient group, the prevalence of ad- verse effects was higher in the out-patient group. This may imply that the in-patient treatment was helpful for decreasing the adverse effects. However, several different explanations can be made for this result and it seems difficult to derive a solid conclusion on which the treatment measure is better. When reasons for the lower incidence of adverse effects in the in-patient group is speculated, first, the in-patient group took sufficient rest, escaping from the daily life, whereas, the out-patient group had to take steroid as performing various routine job-related duties. Due to such difference, the drug metabolism of the patients may have been different between the two groups, and consequently, the prevalence of adverse effects may differ. Secondly, the in-patient group took healthy meal regularly in optimized quantity, as provided by the hospital, whereas, it was impossible for medical staff to control the eating hours, meal quantity and its content in the out-patients group. Thereby, some significant differences are likely to incur in abdominal discomfort felt by patients. As shown in Fig. 5, several differences exist between the 2 groups, but also it was identified that the difference in abdominal discomfort would be the largest. Thirdly, the in-patient group may have had immediate resolution of discomfort before medical record on the adverse effect was made. This is because the patients can meet the medical staff, including doctors and nurses, for 7 days at any time on a daily basis and the medical staff will manage the discomfort more readily than the out-patient group. When immediate symptomatic therapy has been done, patients may not consider the discomfort as a problem anymore and no record may be left. For example, more antacid may have been prescribed in the in-patients group, and this may have reduced the incidence of abdominal discomfort in this group. Lastly, since these two groups were not assigned randomly, it is likely that there is a selection bias. The in-patients were those who had selected to get more active treatment, regardless what the reasons were. For example, they had selected to be admitted to the hospital because either the disease for which steroid had been used, got more aggravated, or due to other past medical history. Accordingly, it may be difficult to make a simple comparison between the two groups. Aforementioned matters are important weaknesses of this study, and because of such, it is difficult to prove whether there is any difference in the actual prevalence of the adverse effects between the in-patient group and the out-patient group. However, given the fact that most adverse effects appear within the first week, the in-patient treatment during the first 1 week may be clinically relevant. In addition, acute management is available when any important adverse effects do occur and this may be an advantage of hospitalized treatment.

Toxic hepatitis and avascular necrosis of hip bone were considered as serious adverse effects. And these serious side ef- 
fects had incurred in 5 patients in total. However, it is unclear whether a short-term steroid prescription has a direct causal relationship with these diseases, or coincidently accompanied otherwise. In particular, 1 patient with toxic hepatitis had died of the disease, but this case also could not be identified whether the cause of death was steroid or not. It was impossible to analyze the risk factors of serious adverse effects because there were not many incidences. But in case of toxic hepatitis, the liver-related baseline disease is considered as an important factor from the fact that the patient was already a carrier of hepatitis virus even before the drug administration or that the hepatitis had incurred from the patient whose serum aspartate transaminase (AST) level or alanine transaminase (ALT) level were already high from the beginning. Therefore, it is considered that we can be prepared against the onset of such serious adverse effects by checking whether the patient is a carrier of hepatitis virus, and by checking the liver function test results in terms of serum AST and ALT levels in advance, before administering the high-dose steroid.

Although the results are interesting, there are several shortcomings that may need careful interpretation to this study. First, due to the retrospective design, we may have neglected some important adverse effects, which cannot be detected by the patient, such as hyperglysemia. Second, in all the patients, antacids were prescribe together with the steroid and in about half of the patients antiviral agents and ginkgo leaf extraction agents were prescribed together with the steroid. Accordingly, some adverse effects may have been due to these adjunctive medications other than steroid. But since adjunctive medications were prescribed in low does and it has been already known that these medications are not related with such adverse effects, we believe that most of the adverse effect is due to steroid. But this point needs further verification.

\section{Conclusion}

The incidence of high dose short term steroid treatment may be very high. The patients should be warned about these adverse effects. Monitoring should be performed until 3-4 week, since some adverse effects may not be detected until this time point.

\section{REFERENCES}

1) Cohn LA. Glucocorticosteroids as immunosuppressive agents. Semin Vet Med Surg (Small Anim) 1997;12:150-6.

2) Rha KS. Otorhinolaryngology-Head and neck surgery. Seoul: Iljogak;2009.

3) Lieberman P, Patterson R, Kunske R. Complications of long-term steroid therapy for asthma. J Allergy Clin Immunol 1972;49:329-36.

4) Chon KM, Goh EK, Lee IW, Cho KS. Clinical observation of sudden deafness in children. Korean J Otolaryngol-Head Neck Surg 2002;45:456-61.

5) Veenstra DL, Best JH, Hornberger J, Sullivan SD, Hricik DE. Incidence and long-term cost of steroid-related side effects after renal transplantation. Am J Kidney Dis 1999;33:829-39.

6) Fahim A, Faruqi S, Wright CE, Kastelik JA, Morice AH. Comparison of the effect of high-dose inhaled budesonide and fluticasone on adrenal function in patients with severe chronic obstructive pulmonary disease. Ann Thorac Med 2012;7:140-4.

7) Kyle V, Hazleman BL. Treatment of polymyalgia rheumatica and giant cell arteritis. II. Relation between steroid dose and steroid associated side effects. Ann Rheum Dis 1989;48:662-6. 